Estudios sobre el Mensaje Periodístico

ISSN-e: 1988-2696

http://dx.doi.org/10.5209/ESMP.55579

\title{
Estudio del Periodismo de Prevención en la prensa diaria. Sustento teórico y análisis de presencia
}

\author{
Silvia Alende Castro ${ }^{1}$; Aurora García González²; José David Urchaga Litago 3
}

Recibido: 29 de enero de 2016 / Aceptado: 27 de junio de 2016

Resumen. Al verse afectado el control sobre el entorno, el riesgo provoca una representación de desorden espacial y temporal. En el caso de la comunicación, la presencia de riesgos llama la atención sobre la capacidad del periodismo para contribuir a la prevención de estas situaciones. Así, en este trabajo se presentan los resultados de una estudio más amplio sobre el modelo teórico en el que se sustenta el Periodismo de Prevención (PdP) así como sobre su presencia en la práctica periodística española, testada a través del análisis de contenido. Metodológicamente, este estudio plantea un análisis de más de 30.000 unidades informativas revisadas en 406 ejemplares de 12 diarios españoles diarios. Entre las principales conclusiones, esta investigación permite afirmar que el PdP cuenta todavía con una escasa presencia en la prensa diaria.

Palabras clave: Periodismo; periodismo de prevención; medios de comunicación; nuevas tendencias de la comunicación.

\section{[en] Study of Prevention Journalism in daily newspapers. Theory and presence analysis}

\begin{abstract}
Concerning the control over the environment, the risk leads to a representation of spatial and temporal disorder. In the case of communication, the presence of risk draws attention to the capacity of journalism to contribute to the prevention of these situations. Thus, this work shows the results of a broader study on the theoretical model of Prevention Journalism (PJ). It is also discussed his presence in Spanish journalistic practice, tested by the analysis of content. Methodologically, this study analized more than 30,000 informational units corresponding to 406 samples of 12 daily Spanish newspapers. Among the main findings, this research allows to assert that the PJ is a trend still with little presence in daily newspapers.
\end{abstract}

Keywords: Journalism; Prevention Jounalism; media; new communication tendency.

Sumario. 1. Introducción y estado de la cuestión. 2. Material y métodos. 3. Análisis y resultados. 4. Conclusiones. 5. Referencias bibliográficas.

Cómo citar: Alende Castro, Silvia; García González, Aurora; Urchaga Litago, José David (2017): "Estudio del Periodismo de Prevención en la prensa diaria. Sustento teórico y análisis de presencia", en Estudios sobre el Mensaje Periodístico 23 (1), 13-27.

1 Universidad de Vigo.

E-mail: silvia.alende@gmail.com

2 Universidad de Vigo.

E-mail: auroragg@uvigo.es

3 Universidad Pontificia de Salamanca.

E-mail: jdurchagali@upsa.es 


\section{Introducción y estado de la cuestión}

En los últimos años, se han explorado propuestas comunicativas vinculadas a nuevos modelos de uso de los medios de comunicación. En este mismo contexto de tránsito de la figura del consumidor al prosumidor (García \& Valdivia, 2014), emerge el estudio de alternativas de la comunicación periodística que plantean contenidos y discursos para el cambio social en el marco de la vida cotidiana y el entorno local. Es el caso del Periodismo de Prevención (PdP). Como modalidad comunicativa que persigue el bienestar social, el amparo teórico considerado más adecuado para el Periodismo de Prevención es la Teoría de la Acción Social, pues "al comprender el objeto como una acción, la ciencia de la comunicación se integra automáticamente en el ámbito de la Ciencia General de la Acción Social” (Román, 2000: 120). Esta teoría tiene entre sus referentes al sociólogo Alfred Schütz, que comprende esta corriente como un método de aproximación a lo cotidiano (Rizo, 2006: 1). Para contextualizar la relación de la Sociología Fenomenológica con la comunicación hay que remitirse a la Escuela de Chicago (Rizo, 2009: 27).

Al respecto, Martín Algarra subraya que "nuestra condición social explica por qué el ser humano se comunica: nuestra naturaleza individual lo exige y nuestra naturaleza social lo hace posible" (2003: 164). Además, la comunicación tiene también un fin: conseguir la armonía entre esas dos facetas, lo individual y lo social (Martín Algarra, 2003: 165). En este punto, se observa cómo el PdP puede actuar como nexo de unión de ambas, pues tiene su eje central precisamente en las personas y en el uso que estas pueden hacer de la comunicación periodística para tratar de evitar ciertas situaciones de riesgo y en las propias capacidades de esta corriente para cumplir esta demanda.

Se entiende por riesgo la percepción que los sujetos tienen al verse afectado el control (cognitivo y práctico) sobre el entorno, lo que provoca una representación de desorden espacial y temporal. Por tanto, una sensación de inseguridad que Piñuel, Gaitán y Lozano han definido con la noción de "quiebras del acontecer" (2013). El riesgo tiene lugar entonces en el momento en que el individuo comprende la posibilidad de que se produzca una ruptura en la estabilidad de su entorno, dando lugar a sorpresa e inestabilidad. Por tanto, el estudio del PdP puede adscribirse también al análisis de los efectos de la comunicación sobre la conducta de los individuos (McQuail y Windahl, 1989: 98), pues en este contexto, "las sociedades modernas desarrollan un típico modelo [...] que provoca riesgos, contingencia y peligro" (Alfie y Méndez, 2000: 174). De acuerdo con Beck, en la modernidad desarrollada "aparece un nuevo destino adscriptivo de peligro, del que no hay manera de escapar" (1998: 12). La llamada sociedad del riesgo (Beck, 1998: 14) advierte procesos como el reparto de la riqueza, la individualización, la globalización o el subempleo. Explica Beck en este sentido que en la modernidad avanzada, la producción social de riqueza va acompañada sistemáticamente de la generación de riesgos. Es decir, el peligro no se deriva ya de la miseria que caracterizaba etapas sociales anteriores sino que en esta era es precisamente el proceso de producción el que entraña conflictos (1998). Los riesgos de la civilización son, según el autor, un barril de necesidades sin fondo, inacabable, infinito y autoinstaurable. Por tanto, el sujeto está siempre afectado por los riesgos, que se presentan de una manera universal y motivan que la ciudadanía esté expuesta a potenciales situaciones de peligro (1998: 29). Coincide, el estudio de esta noción, con la fase del periodismo contemporáneo (Guillamet, 2003: 43), en la que buena parte del imaginario social se funda en representaciones ofrecidas por una 
esfera mediática (Calle, 2005: 179) afectada por procesos de convergencia digital (Navarro, 2009)

Abordar la gestión de riesgos y, en consecuencia, también en este caso la capacidad de prevención de los medios de comunicación implica detenerse en las funciones asociadas a los mismos. Sobre estos quehaceres, existen distintas taxonomías detalladas por autores como Wright (1972), Martínez Terrero (2006), José Luis Martínez Albertos (1972), Denis McQuail (1991 y 1998) o Emil Dovifat (1980). Parte de estas acciones relativas a la labor de informar entroncan con el bien común y la acción en el entorno, pues el fin social de los medios de comunicación puede resumirse como la obligación de las empresas mediáticas de buscar, antes que su propia rentabilidad económica, el beneficio de la sociedad (Tallón, 1992: 19). Una idea que refrendan otros autores como Maciá (2013: 853) o Coca y Peñalva (1998: 23-24). En esta labor emerge la capacidad de los medios de actuar en su propio entorno y permitir anticiparse a ciertas situaciones. Esta tarea orientadora de los medios fue esbozada ya por Wright (1972: 19) y, en los años setenta del pasado siglo, "la sociedad experimentó un giro de tal naturaleza que el énfasis de las noticias empieza a recaer en las preocupaciones sociales" (Diezhandino, 1994: 24). A lo largo del siglo XX fueron apareciendo entonces nuevas formas de informar como un modo de dar respuesta a distintas demandas (López, 2012: 23).

En la anticipación periodística a ciertas circunstancias, enfoque de prevención, se observan distintos antecedentes como son el periodismo de/para la paz (López, 2012: 98) pero también el periodismo cívico o público, nacido contra la apatía de la ciudadanía por las instituciones (Dader, 1999: 16). El periodismo de anticipación, estratégico o de soluciones -impulsado por Claude Monnier como un periodismo que permita al lector resolver problemas (1988)-, el Periodismo Premonitorio (Martínez Muñoz, 2002) o el Periodismo Social —que asume la responsabilidad del periodismo en los procesos sociales (Muñoz, 2011: 27) — son otras modalidades antecesoras. En esta línea, el PdP que aquí se plantea se comprende como heredero, además, de otras dos especialidades periodísticas: el Periodismo de Servicio —del que toma especialmente el concepto de comunicación de utilidad- $-\mathrm{y}$ del Periodismo Preventivo, puesto que reflexionar sobre el Periodismo de Prevención en España exige acudir inevitablemente al trabajo realizado por el Instituto de Periodismo Preventivo y Análisis Internacional (Ippai). Cabe señalar que aquí se ha optado por la denominación Periodismo de Prevención frente a Periodismo Preventivo y es necesario explicar las razones por las que se elige esta denominación en lugar de la más habitual para referirse a la capacidad preventiva de los medios, Periodismo Preventivo. Aunque la propuesta del PdP tiene puntos comunes con el Periodismo Preventivo formulado por el (Ippai), entre ambas conceptualizaciones también hay diferencias - o al menos disparidades concretas - , por lo que Periodismo de Prevención parece una denominación más amplia y adecuada en este caso. Amplia porque, frente al Periodismo Preventivo - centrado en grandes crisis y conflictos solo en el ámbito internacional (Bernabé, 2007) — el PdP extiende su punto de mira a cualquier territorio, por pequeño que sea, en el que se perciban posibilidades de prevención desde la perspectiva mediática. Es decir, para el PdP lo importante no es el número o la extensión afectada por algún desastre sino la posibilidad de ayudar a alguien a evitar un riesgo. Se entiende también que se trata de una denominación más justa, en el sentido de que plantear puntos divergentes frente a la tendencia propuesta por el Ippai exige, para resultar más claros, identificar los presupuestos propios con una corriente concreta, evitando la confusión de cada uno de estos dos postulados. Finalmente, 
Periodismo de Prevención semeja un concepto más adecuado al planteamiento que aquí se propone, precisamente por ser la amplitud lo que define a este enfoque.

Como se avanzó, el PdP se caracteriza además por el concepto de comunicación de utilidad, estudiado en el marco del Periodismo de Servicio. Existe en el ámbito periodístico una discusión acerca de si todo el periodismo es o no útil. Así, para Alberdi, Armentia, Caminos y Marín "cualquier actividad periodística, el periodismo en sí mismo, ya que se trata de la misma esencia del periodismo, es o debe ser útil para el público en general o para algún sector del público en particular" (2002: 69). Esta opinión la refrendan otros autores como MacDougall, que afirma que "el periódico no tiene otra razón de existir que el servicio público" (Diezhandino, 1994: 75). Sin embargo, se acepta aquí que la utilidad puede ser entendida y buscada en el periodismo de modo mucho más concreto, al comprenderse específicamente esta característica como un ingrediente muy concreto de ciertas modalidades periodísticas. Como rasgo distintivo, la comunicación útil presenta una intención más profunda de ayuda al lector, de contribución positiva a su vida (Diezhandino, 1994: 23). En este sentido, se establecen distintos grados de utilidad. De este modo, se podría hablar de una primera utilidad (Grado de Utilidad 1) en el caso de la información utilizable en el sentido de hágalo usted mismo. Se trata de una "vieja práctica periodística" (Diezhandino, 1994: 93) influenciada por "nuevos hábitos sociales". Es un tipo de utilidad, valga la redundancia, útil y provechosa. No obstante no podría considerarse, en general, un contenido vital, sino complementario en la vida del lector. En segundo lugar (Grado de utilidad 2), en función de los estudios de Diezhandino, podría englobarse en este eslabón todo aquel contenido que trata de influir, de alguna manera, en el receptor ofreciéndole pautas de vida o referentes a tener en cuenta. Así, la autora ve utilidad por ejemplo en la información que impulsa a actuar en un sentido determinado y cita por ejemplo la promoción de campañas a favor de determinadas causas sociales (Diezhandino, 1994: 94-96). En el caso del Grado de utilidad 3, nivel con el que se identifica especialmente el Periodismo de Prevención, se hace alusión al contenido informativo que permita actuar, en la medida de lo posible, a favor de la prevención de situaciones de riesgo. Este tipo de utilidad se vincula con la identificada por Pilar Diezhandino (1994: 94) con la información que induce a prevenir un mal que se presenta como posibilidad. Se trata de evitar, en la medida de lo posible, que esa situación negativa se alcance. Por tanto, la relación de este tipo de datos con el bienestar y la propia vida del ser humano es estrecha. No se trata de datos que busquen mitigar, calmar o suavizar un determinado estado o circunstancias mejorables. Es un paso anterior, en el que la comunicación intenta ser útil para el lector del modo más vital, el del mantenimiento del propio orden de seguridad (Eide y Knight, 1999).

Por tanto, y en consonancia con la idea propuesta por Xosé López de que "otro periodismo es posible" y de que "solo queda, pues, intentar reflexionar cómo hacerlo y establecer las estrategias adecuadas" (2012: 12), se detalla el planteamiento teórico sobre el Periodismo de Prevención que se sugiere en esta investigación: un periodismo que tiene en la ética su punto de partida, en la anticipación su principal atributo y en las situaciones de riesgo su enfoque. Conceptualmente, esta perspectiva busca englobarse en el Periodismo Total, pues trata de "mostrar caminos innovadores que aportan soluciones a los problemas de la sociedad” (López, 2012: 169).

En este modelo, se entiende que si informar es una obligación de los medios, hacerlo desde una perspectiva de prevención se presenta entonces como una actividad informativa con evidentes repercusiones éticas (Diezhandino et al., 2002: 9). Esta 
idea de cooperación es objeto de investigación en medios (Aznar, 2004), fundamentalmente a través de estudios sobre la comunicación para el desarrollo y la solidaridad, tal y como detallan autores como Canela (2007) o Graziano (1997). En consonancia con González Álvarez, la información "debería ser un proceso volcado a proporcionar a los individuos el poder del conocimiento para que tomen decisiones reflexivas con el fin de mejorar sus vidas y el conjunto de la sociedad" (2012: 241). Según la concepción que aquí se plantea, el periodista que practica el PdP pone al servicio de la ciudadanía sus capacidades técnicas para transmitir información pero sobre todo su responsabilidad profesional, moral y social (Unesco, 1983). Esta tendencia supone por tanto una intencionalidad, puesto que el PdP no busca existir a la deriva de la actualidad sino que sin perderla de vista pretende "ir más allá como producto de una postura deliberada de los medios" (Obregón et al., 2010: 132).

Por tanto, el PdP puede ser entendido además como uno de los retos del periodismo actual. De hecho, Diezhandino identifica el valor añadido de los medios en la actualidad precisamente con la capacidad de guiar, orientar y hacer prospecciones (2012: 49). Junto a la ética, en esta propuesta teórica se acepta la anticipación como otro de los principales atributos del PdP. Para la argentina Luz Marina Mateo, la anticipación "implica tomar en cuenta aquellos sucesos que, aunque parezcan aislados o inconexos, pueden llevar a que se desate un conflicto" (2010). El concepto de anticipación suele vincularse, en la investigación periodística, a la cobertura de desastres, especialmente de tipo natural, tal y como han analizado autores como Morales (2006), Bernabé (2007), Obregón, Arroyave y Barrios (2010), Soria (1997), Miralles (2009) o González Borjas (2004). Pero la anticipación se entiende además como un servicio, pues "estando alertada la población puede movilizarse y evitar su destrucción" (Wright, 1972: 19).

Por otra parte, la anticipación periodística no suele concebirse como un valornoticia o criterio noticiosos, en las taxonomías planteadas por estudiosos como Quesada (2007), Goiricelaya (1998), Muñoz Torres (2002) o Moreno, Rovetto y Buitrago (2007). Por tanto, el Periodismo de Prevención tal y como aquí se plantea convierte en valores noticia criterios hasta ahora dejados al margen. Sin dejar de prestarles atención, supone y exige, entonces, una revisión de los valores noticiosos clásicos. Así, será la "responsabilidad por el otro" (Martínez Antón, 2004: 214) el criterio que, en Periodismo de Prevención (el papel previsor del periodismo se ha vinculado internacionalmente a la gestión de riesgos ${ }^{4}$ ) ha de validar el contenido de la noticia de prevención. En definitiva, el PdP puede definirse como aquella práctica periodística que, a través de una actitud consciente, busca aproximarse a la cotidianeidad del lector por medio de la publicación de información de carácter útil que permita una adecuada gestión de riesgos en su entorno próximo. Con la prevención como objetivo primordial, esta tendencia que retoma los valores éticos de la profesión y reformula los criterios de noticiabilidad.

\section{Material y métodos}

Tras la exposición de la conceptualización teórica en la que se asienta este trabajo, se presenta la investigación aplicada realizada para el análisis del Periodismo de Prevención en la prensa diaria. Este estudio nace del interés por conocer si en la prensa

$4 \quad$ Véase por ejemplo Cenapred (s/f), Unisdr (2009), Morales (2006), Villalobos (1998) o Bernabé (2007) 
editada con periodicidad diaria se practica el PdP pero, además, se propone convertirse en una llamada de atención sobre las características, particularidades y fines de esta tendencia periodística. La novedad de la temática aquí planteada se entiende como una de las principales características de esta investigación. Hasta el momento, no se han encontrado estudios similares ${ }^{5}$. Así, se planteó una hipótesis principal y tres secundarias, con la pretensión de su posterior corroboración.

Hipótesis 1: El Periodismo de Prevención está representado en los medios de comunicación editados con periodicidad diaria. Esta representación es, sin embargo, todavía escasa.

Hipótesis 1a: El PdP obedece más a una finalidad genérica de control del entorno que a la oferta de pautas claras de actuación para el lector.

Hipótesis 1b: Los contenidos que nutren el PdP presentan un alto grado de utilidad.

Hipótesis 1c: El PdP que se practica actualmente responde todavía a una fase previa de esta corriente y supone más una puesta en guardia del público que una prevención en sí misma.

Esta hipótesis principal — junto a las tres secundarias - hace referencia a un aspecto concreto del PdP, la cantidad. Metodológicamente, se ha optado por el análisis de contenido como un enfoque de investigación empleado con frecuencia en todas las áreas relacionadas con los medios, puesto que esta técnica de investigación permite "realizar inferencias válidas y estables (replicables) a partir de unos datos, en torno a su contexto" (Wimmer y Dominick, 1996: 170) al estudiar cuantitativamente material cualitativo no estructurado o libre (Urchaga, 2009), en este caso mensajes informativos (Bergh, 1989: 105)

Se estudiaron un total de 12 diarios generalistas y de pago españoles: El Correo Gallego, La Región, El Progreso, Diario de Pontevedra, Diario de Arousa, Diario de Ferrol, El Ideal Gallego-Diario de Bergantiños, Atlántico Diario, La Opinión de A Coruña, La Voz de Galicia y Faro de Vigo. En estos dos últimos casos, se incluyeron en la muestra de análisis los ejemplares correspondientes a cada una de las ediciones de ambas cabeceras: La Voz de Galicia (A Coruña, A Mariña, Arousa, Barbanza, Carballo, Deza, Ferrol, Lemos, Lugo, Ourense, Pontevedra, Santiago y Vigo) y Faro de Vigo (Arousa, Deza, O Morrazo, Pontevedra, Ourense y Vigo). La muestra suma, en conjunto, 29 ediciones diferentes por cada día de estudio. Estos periódicos fueron revisados durante 14 meses. Se recuperaron, en concreto, los ejemplares correspondientes a las siguientes fechas: 14 de noviembre de 2012, 15 de noviembre de 2012, 15 de diciembre de 2012, 15 de enero de 2013, 15 de febrero de 2013, 15 de marzo de 2013, 15 de abril de 2013, 15 de mayo de 2013, 15 de junio de 2013, 15 de julio de 2013, 15 de agosto de 2013, 15 de septiembre de 2013, 15 de octubre de 2013, 15 de diciembre de 2013. Cabe matizar que la primera intención de esta investigación consistía en analizar cada uno de los diarios publicados en Galicia el día 15 de cada mes durante el año 2013. Con esta determinación, se pretendía, partiendo de una muestra aleatoria, contar con un volumen de material lo suficientemente representativo del fenómeno objeto de estudio. Según estos parámetros, esa primera selección quedaba conformada del siguiente modo:

- Dos lunes: 15 abril y 15 de julio

— Dos martes: 15 de enero y 15 de octubre

Se han consultado CSIC, Dialnet y Teseo. 
- Un miércoles: 15 de mayo

- Un jueves: 15 de agosto

- Tres viernes: 15 de febrero, 15 de marzo y 15 de noviembre

— Un sábado: 15 de junio

— Dos domingos: 15 de septiembre y 15 de diciembre

Sin embargo, con el objetivo de obtener la misma representatividad por cada día de la semana, se decidió posteriormente ampliar esta muestra a 14 días, sumados dos de los meses de noviembre y diciembre de 2012. De este modo, se eliminó un viernes de 2013 (había 3) y se optó por introducir un miércoles, un jueves y un sábado más de la tercera semana de noviembre y diciembre de 2012. En concreto, se eligieron el miércoles 14 de noviembre de 2012 (es el más cercano al 15), el jueves 15 de noviembre de 2012 y el sábado 15 de diciembre de 2012. A nivel temporal, la muestra final quedó conformada, entonces, del siguiente modo:

— Dos lunes: 15 abril y 15 de julio de 2013

- Dos martes: 15 de enero y 15 de octubre de 2013

- Dos miércoles: 15 de mayo de 2013 y 14 de noviembre de 2012

- Dos jueves: 15 de agosto de 2013 y 15 de noviembre de 2012

— Dos viernes: 15 de febrero y 15 de marzo de 2013 (se eliminó el 15 de noviembre, por contar con mayor representación de este mes correspondiente a 2012)

— Dos sábados: 15 de junio de 2013 y 15 de diciembre de 2012

— Dos domingos: 15 de septiembre y 15 de diciembre de 2013

Por, tanto, se analizaron 406 ejemplares (29 ediciones por 14 días) y más de 30.000 unidades informativas. Una vez se determinó el material de análisis, se inició la fase de identificación de aquellas unidades informativas que podían vincularse a la tendencia Periodismo de Prevención y de la catalogación de estas unidades resultó el universo de estudio definitivo al que se aplicó el análisis de contenido propuesto. A la hora de discernir, en cada uno de los periódicos analizados, si una información podía considerarse o no como PdP, se tomaron en cuenta los siguientes criterios de identificación concretos, derivados de la revisión y el planteamiento teórico realizados para el PdP:

A. Se derivaron de una intención evidente del periodista por tomar posición respecto al bienestar del público.

B. Incidieron desde una perspectiva de vigilancia o control, en elementos de riesgo.

C. Valoraron la existencia de protocolos y su idoneidad.

D. Llamaron la atención sobre asuntos que solían causar situaciones de riesgo.

E. En el caso de derivarse de un suceso reciente, no se redujeron al mero relato de esos hechos sino que avanzaban en su comprensión proponiendo soluciones útiles.

F. Guías: ofrecieron indicaciones.

G. Buscaron, de manera clara e inequívoca, anticipar, avisar, alertar o posicionar al lector.

Una vez aplicados estos criterios a la revisión íntegra de los 406 ejemplares seleccionados, se logró identificar un total de 150 unidades informativas que respondían a criterios de Periodismo de Prevención. Su estudio en detalle se llevó a cabo mediante 
un análisis de contenido para el que se utilizó como instrumento de codificación un code-book compuesto por las siguientes variables relativas, en este caso, a la cantidad de PdP: cabecera (identificación del periódico en el que se publicó cada unidad informativa), temporalización (estudio de la fecha de publicación), día de la semana (estudio de la jornada semanal de publicación), género (estudio del género de publicación: noticia, reportaje, crónica, entrevista, editorial, otros) grado de utilidad de la información (grado I, II o III) ${ }^{6}$ y fase de prevención. En este último caso y tras una primera revisión de contenidos, se establecieron las siguientes como posibles fases de prevención:

1. Periodismo de Prevención-Óptimo: piezas informativas que cumplían con claridad alguno de los criterios de inclusión.

2. Periodismo de Prevención en fase de alerta: piezas informativas que, aun cumpliendo alguno de estos criterios, no ofrecían claramente datos de prevención pero sí constituyen una alerta.

3. Llamada de atención a la Administración: piezas informativas que, pese a no ser constituyentes de acciones preventivas en sí mismas, sí podían servir como medio para que la Administración competente en una determinada materia actuase a favor de la prevención.

4. Antesala de la prevención: piezas informativas que se aproximaban a alguna de las situaciones descritas en los criterios preventivos pero sin llegar a cumplir en su totalidad alguno de ellos.

5. Prevención implícita: piezas informativas que, sin ofrecer claramente contenidos preventivos, sí contenían algunos datos para que el lector pudiese anticiparse.

Detallada la metodología empleada para el análisis empírico de esta investigación, a continuación se presentan sus resultados.

\section{Análisis y resultados}

En este apartado se presentan resultados de carácter cuantitativo correspondientes al estudio de las hipótesis principal y secundarias de investigación, así como a los problemas exploratorios planteados conjuntamente. Estos datos hacen referencia al conjunto de todas las unidades (fueron tratados con el programa informático SPSS 18.0.) y son referentes al aspecto cuantitativo de lo publicado — cantidad —, que se abordó, en primer lugar, desde el estudio de la presencia de esta perspectiva periodística así como el número de criterios de inclusión que suele reunir. Por otro lado, interesó además describir dicha presencia según la cabecera, el día de la semana, la temporalización y el género periodístico. Por último se diferenció en función del grado de utilidad así como de la fase de prevención a la que hace referencia el contenido.

Para testar la hipótesis general se ha comparado el número de unidades de Periodismo de Prevención (150) con el número de ejemplares y con el número de unidades informativas totales presentes en los ejemplares analizados. En el total de cabeceras analizadas fueron contabilizadas 31.545 unidades informativas, oscilando

Según el planteamiento teórico relativo a la gradación de utilidad. 
entre las 8.515 unidades de La Voz de Galicia y las 1.518 de La Región (ver gráfica 1.). Estos datos mostraron que el número de unidades de PdP halladas es muy bajo (150) ya se compare con el total de unidades (31.545) o con el número de ejemplares (406). En el primer caso, la proporción no alcanzó ni el 1\% de las unidades totales, siendo el número aproximado de 4,8 por cada 1.000 unidades analizadas. Esta proporción fue (aunque muy desigual) muy baja en todas las cabeceras.

Si se comparan las unidades de PdP con el número de ejemplares analizados se observa que no se alcanzó ni una unidad (150) por ejemplar (406). Es decir, aproximadamente hay que leer casi tres ejemplares para poder encontrar una unidad de PdP (la media exacta es 2,71). Por lo tanto esta hipótesis queda confirmada, ya que las unidades de PdP halladas fueron una minoría (150) tanto en comparación con los ejemplares (406) como con el total de unidades (31.545) pero se pudo comprobar, al tiempo, que el PdP está representado en la prensa diaria.

Tabla. 1. Presencia de Periodismo de Prevención. Elaboración propia.

\begin{tabular}{lccccc}
\hline \multicolumn{1}{c}{ Cabecera } & Ejemplares & $\begin{array}{c}\text { No Unidades } \\
\text { Informativas }\end{array}$ & No PdP & $\begin{array}{c}\text { No PdP } \\
\text { (cada 1.000) }\end{array}$ & $\begin{array}{c}\text { No PdP } \\
\text { (por ejemplar) }\end{array}$ \\
\hline Diario de Pontevedra & 14 & 1.773 & 25 & 14,1 & 1,8 \\
El Progreso & 14 & 2.032 & 24 & 11,8 & 1,7 \\
El Correo Gallego & 14 & 1.938 & 14 & 7,2 & 1,0 \\
Faro de Vigo & 84 & 5.397 & 34 & 6,3 & 0,4 \\
Opinión de A Coruña & 14 & 1.800 & 11 & 6,1 & 0,8 \\
La Región & 14 & 1.518 & 8 & 5,3 & 0,6 \\
Diario de Arousa & 14 & 1.607 & 8 & 5,0 & 0,6 \\
Atlántico Diario & 14 & 1.670 & 7 & 4,2 & 0,5 \\
Diario de Bergantiños & 14 & 1.753 & 3 & 1,7 & 0,2 \\
La Voz de Galicia & 182 & 8.515 & 12 & 1,4 & 0,1 \\
Diario de Ferrol & 14 & 1.788 & 2 & 1,1 & 0,1 \\
El Ideal Gallego & 14 & 1.754 & 2 & 1,1 & 0,1 \\
\hline Suma $\left(\sum\right)$ / Media $(\overline{\mathrm{X}})$ & $\sum: 406$ & $\sum: 31.545$ & $\sum: 150$ & $\overline{\mathrm{X}}: 4,8$ & $\overline{\mathrm{X}}: 0,4$ \\
\hline
\end{tabular}

En el contexto de esta hipótesis, se plantearon además varios problemas de investigación —como hipótesis secundarias - referentes a la presencia cuantitativa del PdP. La primera cuestión se vinculó al cumplimiento de los siete criterios de inclusión vistos por cada unidad. De ellos, el B (Vigilancia de la normalidad) fue el requisito más presente $(35,3 \%)$, seguido del $\mathrm{G}$ (anticipación y posicionamiento del lector) con un $25,8 \%$ y del C (valoración del cumplimiento de protocolos: $17,2 \%$ ). Se trata, claramente, de los tres criterios más habituales en las unidades de prevención, que en mucha menor medida también aportaron soluciones a un hecho concreto (E: 6,6\%), alertaron de posibles accidentes habituales (D: 6,1\%), sirvieron de guía (F: 5\%) o se derivaron del ánimo evidente del periodista por prevenir (A: 4\%). Por tanto, se observa que el PdP atiende más a una finalidad genérica de control del entorno y de puesta en guardia del lector. Sin embargo, se encuentra más alejado de la búsqueda de soluciones a partir 
de hechos de la propuesta de guías o del ofrecimiento al usuario de la información de pautas claras de actuación, confirmándose así la hipótesis 1a.

Tabla 2. Índice de cumplimiento de criterios de prevención. Elaboración propia.

Criterio

B. Vigilancia de la normalidad

G. Anticipación y posicionamiento del lector

C. Valoración de protocolos

E. Soluciones a partir de un hecho reciente

D. Accidentes (intoxicación, etc.)

F. Guías

A. Posición del medio/periodista
Frecuencia (n) Porcentaje (\%)

86

35,3

63

25,8

42

17,2

16

6,6

15

6,1

12

5,0

10
4,0

\begin{tabular}{lll}
\hline Suma $(\Sigma)$ & $\sum: 150 \quad \sum: 100 \%$
\end{tabular}

Tras analizar cuál fue el orden de cumplimiento de estos siete criterios, pareció interesante cuantificar además la presencia de estos requisitos por unidades. Así, se observó que 74 de las 150 unidades de PdP analizadas cumplían un solo requisito. Por tanto, el índice de inclusión de criterios fue bajo, puesto que en un 49,3\% de los casos se cumplía un solo criterio o dos (40\%). De media, se cumplieron 1,63 requisitos por unidad y, como mucho, se constataron cuatro criterios en una misma noticia (dos casos). En ningún caso se cumplieron 5 o más criterios. Estos datos permitieron comprobar un reducido índice de cumplimiento de criterios, parámetros que pueden identificarse con el propio objetivo de esa información de prevención.

En cuanto al día de la semana, se observó que hay diferencias a la hora de hallar PdP en la prensa. Así, lo más frecuente fue que las unidades de prevención apareciesen los miércoles $(22 \%)$ o los viernes $(21,3 \%)$. Los martes y los jueves fueron, con un $17,3 \%$ y un $14,7 \%$, respectivamente, las siguientes jornadas en las que se detectó la presencia de esta tendencia. Sábados $(10 \%)$, lunes $(9,3 \%)$ y domingos $(5,3 \%)$ fueron los días en los que la frecuencia fue menor. De este modo, se puede deducir que la publicación de $\mathrm{PdP}$ no parece guardar relación con los ritmos de lectura de prensa comúnmente aceptados (el 11,5\% de la población solo lee periódicos los fines de semana, INE, 2014) y según los cuales el día de mayor índice de lectura es el domingo. Esta es, sin embargo, la jornada en la que claramente menos PdP se halla. Esta realidad se presenta por tanto contraria a las características de consumo de prensa, tal y como sostienen Gurrea y Favián al afirmar que "la lectura de periódicos en papel suele efectuarse principalmente en fin de semana (69,2\%)" (2008: 117). Respecto a la temporalización, la tendencia que mostraron los datos es una disminución clara de PdP durante el último año, dentro de la tónica general de la poca presencia de este tipo de unidades. Es decir, el volumen de PdP en los medios analizados tiende a la bajada, irregular y con picos concretos en algunos meses como fueron marzo, mayo y octubre de 2013. 
En cuanto al género - la especificación de los géneros periodísticos es esencial para poder determinar sus características discursivas así como su función y estructura (Gutiérrez Vidrio, 2010) - el más frecuente fue la noticia con 120 unidades (80\%), seguido del reportaje con otras $25(16,7 \%)$ y la entrevista con $5(3,3 \%)$. No se hallaron crónicas ni editoriales. Por tanto, se observa claramente el predominio de géneros informativos sobre los interpretativos o de opinión, "unas modalidades discursivas que producen orden en el material informativo [...] para recoger la complejidad de lo que acontece y exponerlo a los receptores" (Fernández Parrat, 2001). Destaca, por ejemplo, la ausencia de crónicas de prevención así como de editoriales. En este último caso, la inexistencia de este género parece síntoma de la falta de interés, todavía, de los medios por incluir una actitud preventiva en estas piezas referentes de la identidad de cada cabecera mediante una tipología del discurso consistente en un hecho provocado por el periódico con la intención de intervenir en la vida social y con el que "trata de modificar una situación" (Gomis, 1974: 318).

En relación al grado de utilidad, no se detectaron informaciones correspondientes al grado de utilidad I, identificado en esta investigación con el Periodismo de Servicio. De los dos grados vinculados al PdP, el II y el III, primó el tres (el más ligado al orden vital de la sociedad) con 83 unidades $(55,3 \%)$ seguido del dos - caso de otras 67 unidades $(44,7 \%)$ - , tal y como se especificaba en la propuesta teórica. Estos datos mostraron, por tanto, que el PdP está muy asociado a contenidos de gran utilidad, como se especificaba en la hipótesis $1 \mathrm{~b}$.

Finalmente, respecto a la fase se pudo comprobar la hipótesis $1 \mathrm{c}$ al observar que de las 150 unidades de PdP analizadas, solo 64 (un 42,7\%) se identificaban claramente con PdP en fase óptima (entendido como aquel que aporta al lector datos que, de modo anticipado, le permiten adquirir conocimiento sobre la gestión de situaciones de potenciales riesgos). Sin embargo, otras 86 (un 57,3\%) se aproximaban de alguna manera a este nivel pero todavía en una fase previa. Es el caso de 47 unidades correspondientes a llamadas de atención a la Administración, de 19 que ofrecían datos de prevención de manera implícita, de otras 15 que constituían una alerta y de 5 identificadas como antesala de la prevención. Por lo tanto, como se señaló al analizar su presencia, el PdP no es fácil de localizar en la prensa diaria.

En resumen, tal y como se pronosticaba, las unidades informativas de prevención son una minoría. Cuando aparecen, lo más probable es que lo hagan en miércoles o viernes. Se presentan sobre todo en noticia, grado de utilidad III (contenidos muy cercanos al orden vital) y realmente como una fase previa a la propia prevención, poniendo en guardia pero no exactamente previniendo. Por lo tanto, se confirma la hipótesis de partida según la cual el PdP posee escasa representación en los periódicos editados con periodicidad diaria así como las tres hipótesis secundarias relativas a las características cuantitativas de esta perspectiva periodística. Al mismo tiempo, esta investigación sienta las bases teóricas de esta corriente periodística enfocada a la prevención, de aplicación en cualquier territorio en el que se detecten riesgos.

\section{Conclusiones}

Una vez finalizado el análisis de PdP en los 406 ejemplares revisados y demostrada la veracidad de las hipótesis planteadas se ofrecen las aportaciones más relevantes de esta investigación: 
Primera. El PdP cuenta todavía con una escasa representación en la prensa diaria pero está presente. Encontrar unidades informativas que respondan a criterios de prevención en estas cabeceras requiere la lectura de cerca de tres ejemplares completos para el hallazgo de un solo contenido de esta clase.

Segunda. Por tipología, el PdP Óptimo es verdaderamente escaso, puesto que en la mayor parte de las ocasiones los contenidos de prevención responden todavía a fases previas de esta corriente.

Tercera. A nivel temporal, la presencia de PdP en la prensa se caracteriza por la variabilidad tanto desde la perspectiva semanal como mensual.

Cuarta. El PdP se practica de manera preferente a través de la noticia como género y en menor medida por medio del reportaje. Entrevistas, crónicas y editoriales no se emplean o son minoritarios.

Quinta. Los contenidos que nutren el PdP que se practica en la actualidad presentan un grado alto de utilidad. Se trata de datos transversales para la ciudadanía.

Sexta. El escaso PdP que se publica se hace siguiendo un número reducido de criterios de prevención. Estas unidades responden más a parámetros de carácter genérico que a un objetivo consciente de ayuda al lector.

En resumen, el PdP es todavía una perspectiva periodística escasamente estudiada y practicada. A diario, los periódicos analizados incluyeron en su agenda temática realidades de riesgo para la ciudadanía que, sin embargo, apenas recibieron un tratamiento de prevención. La cobertura más frecuente de este tipo de sucesos olvida la capacidad preventiva como una posibilidad de los medios y se centra en la mera descripción de lo ocurrido, sin plantearse si este tratamiento puede indicar a los lectores cómo proceder en caso de que una situación de riesgo se repita o sobre cómo evitarla. Por estas razones, esta investigación busca acercarse a los medios y los profesionales con intención de que, de un modo proactivo y anticipado, se asuma un compromiso profesional de ayuda al lector.

\section{Referencias bibliográficas}

Alberdi, Aintzane; Armentia, José Ignacio; Caminos, José María \& Marín, Flora (2002): El diario de servicios en España. Oviedo, Septem.

Alfie, Miriam \& Méndez, Luis (2000): "La sociedad del riesgo: amenaza y promesa". Sociológica, 43, 173-20, en: http://goo.gl/e4JQsH. [Recuperado: 11 de enero de 2015]

Aznar, Hugo (2004): "Los códigos éticos del periodismo y la responsabilidad de los profesionales". Razón y Palabra, 40, en: http://goo.gl/klIPQ [Recuperado: 12 de mayo de 2013]

Beck, Ulrich (1998): La sociedad del riesgo. Hacia una nueva Modernidad. Barcelona, Paidós Ibérica.

Bernabé, Javier (2007): Periodismo preventivo. Otra manera de informar sobre las crisis y los conflictos internacionales. Madrid, Los Libros de la Catarata.

Bergh, Bruce (1989): Qualitative Research methods for the social sciences. Massachussetts: Allyn and Bacon, Needham Heights.

Calle, Ángel (2005): Nuevos movimientos globales. Hacia la radicalidad democrática. Madrid, Editorial Popular.

Canela, Guilherme (2007): "Cubriendo políticas públicas sociales: la importancia de la prensa en los procesos de desarrollo". Seminario Latinoamericano Sin Comunicación 
no hay Desarrollo de la Asociación de Comunicadores Sociales, Lima, en http://goo.gl/ GGaoPY [Recuperado: 16 de febrero de 2015]

Centro Nacional de Prevención de Desastres (CENAPRED) (s/f): Desastres. Guía de Prevención, en: http://goo.gl/5h1bRJ [Recuperado: 10 de febrero de 2015]

Coca, César y Peñalva, José Luis (1998): Modelos de los medios de comunicación social. Periodismo escrito. Zarautz, Servicio Editorial del País Vasco.

Dader, José Luis (1999): "La recuperación ciudadana de los medios: vías de participación y contrapeso crítico de los consumidores y usuarios ante los medios de comunicación de masas”. En VV.AA. Los usuarios de la sociedad de la información. Madrid, Ceaccu, en: http://goo.gl/NYas3n [Recuperado: 11 de abril de 2013]

Diezhandino, Pilar (1994): Periodismo de Servicio: la utilidad como complemento informativo en Time, Newsweek y U.S. News and World Report, y unos apuntes del caso español. Barcelona, Bosch.

Diezhandino, Pilar (Coord., 2012): El periodista en la encrucijada. Madrid, Fundación Telefónica, en: http://goo.gl/2mC2tV [Recuperado: 18 de febrero de 2015]

Diezhandino, Pilar; Marinas, José Miguel; y Watt, Ninfa (2002): Ética de la comunicación, problemas y recursos. Madrid, Edipo.

Dovifat, Emil (1980): Política de la información. Navarra, Eunsa.

Fernández Parratt, Sonia (2001): “El debate en torno a los géneros periodísticos en la prensa: nuevas propuestas de clasificación”. ZER Revista de Estudios de Comunicación, 11, 293-310, en: http://goo.gl/P5DS0N [Recuperado: 19 de enero de 2015]

García, Maricarmen y Valdivia, Angharad (2014): "Prosumidores mediáticos. Cultura participativa de las audiencias y responsabilidad de los medios". Comunicar, 43, 10-13. http://dx.doi.org/10.3916/C43-2014-a2

Goiricelaya, Ernesto (1998): Desastres y medios de comunicación (El caso de Biescas. Agosto de 1996). Asociación Profesional de Técnicos Bomberos: Emergencia 112.

Gomis, Lorenzo (1974): El medio media: la función política de la prensa. Madrid, Seminarios y Ediciones.

González Álvarez, Marta Isabel (2012): "La profesionalización de la Comunicación para la Solidaridad: diagnóstico y propuestas para el sector en España". CIC Cuadernos de Información y Comunicación, 17, 239-253, en: de http://goo.gl/5nbFOp [Recuperado: 21 de agosto de 2013]

Graziano, Margarita (1997): Concepciones del Desarrollo. Material de cátedra.

Guillamet, Jaume (2003): "Por una historia comparada del periodismo. Factores de progreso y atraso". Doxa, 3, 35-56, en http://goo.gl/jvJfzE [Recuperado: 17 de noviembre de 2014]

González Borjas, Antonia (2004): "Salud, información periodística especializada en alza". Ámbitos 11-12, 301-310, en: http://www.redalyc.org/articulo.oa?id=16801216 [Recuperado: 7 de febrero de 2015]

Gurrea, Raquel \& Favián, Carlos (2008): "La lectura de prensa". En Millán, J.A. (Coord.): La lectura en España. Informe 2008: leer para aprender, en: http://goo.gl/UC47qS [Recuperado: 14 de enero de 2015]

Gutiérrez Vidrio, Silvia (2010): "Discurso periodístico: una propuesta analítica". Comunicación y sociedad, 14, 169-198, en: http://goo.gl/watHzy [Recuperado: 19 de enero de 2015]

INE [Instituto Nacional de Estadística, España] (2014): Personas según el hábito de lectura de prensa diaria por nivel de estudios, situación laboral y frecuencia, en: http://www. ine.es/jaxi/tabla.do [Recuperado: 15 de enero de 2014] 
López, Xosé (2012): Movimientos periodísticos. Las múltiples iniciativas profesionales y ciudadanas para salvar los elementos básicos del periodismo en la era digital. Salamanca, Comunicación Social, Ediciones y Publicaciones.

Maciá, Carlos (2013): “Ética periodística, I+D+I y transferencia del conocimiento". Estudios sobre el Mensaje Periodístico, 19, 853-862, en: http://goo.gl/81K0JZ [Recuperado: 28 de enero de 2014]

Martín Algarra, Manuel (2003): Teoría de la comunicación: una propuesta. Madrid, Tecnos. Martínez Albertos, José Luis (1972): La información en una sociedad industrial. Madrid, Editorial Tecnos.

Martínez Antón, Miguel (2004): El periodista, palabra profética en la acción informativa. Reivindicaciones en un contexto mediático. Contenidos del Congreso Internacional de Ética y Derecho de la Información: Información, libertad y derechos humanos: la enseñanza de la ética y el derecho de la información, Valencia, febrero de 2003, en http:// goo.gl/DC2RpY [Recuperado: 20 de octubre de 2014]

Martínez Muñoz, Yolanda (2002): "El periodismo preventivo. El papel de los mass media en la prevención de conflictos armados". Estudios Internacionales de la Complutense, 4, 149-171, en: http://goo.gl/s7Wr8x [Recuperado el 12 de febrero de 2015]

Martínez Terrero, José (2006): Teorías de comunicación. Guayana, Universidad Católica.

Mateo, Luz Marina (2010): "El rol de los medios de comunicación locales en el ejercicio del periodismo preventivo en la Argentina actual un aporte a la comunicación para el desarrollo". Anuario, Instituto de Relaciones Internacionales (IRI), Universidad de La Plata, en: http://goo.gl/wpCPU5 [Recuperado: 18 de mayo de 2013]

McQuail, Denis (1998): La acción de los medios. Los medios de comunicación y el interés público. Buenos Aires, Amorrotu Editores.

McQuail, Denis (1991): Introducción a la teoría de la comunicación de masas. Barcelona, Paidós.

McQuail, Denis \& Windahl, Sven (1989): Modelos para el estudio de la comunicación colectiva. Pamplona, Ediciones Universidad de Navarra.

Miralles, Ana María (2009): Periodismo público en la gestión del riesgo. Lima, Pull Creativo. Monnier, Claude (1988): "El nuevo periodismo de anticipación”. Nuestro Tiempo, 88, 50-59.

Morales, Carlos (2006): Periodistas por la gestión del riesgo de desastres. Costa Rica, CRID, en: http://goo.gl/8dxmND [Recuperado: 8 de abril de 2013]

Moreno, Amparo; Rovetto, Florencia; y Buitrago, Alfonso (2007): ¿De quién hablan las noticias? Guía para humanizar la información. Barcelona, Icaria.

Muñoz, Jhoana Marcela (2011): Periodismo Social, desde la gente, para la gente. Informe de práctica empresarial para optar el título de Comunicadora y Periodista, Corporación Universitaria Lasallista, en: http://goo.gl/hDu2Zp [Recuperado: 3 de noviembre de 2014]

Muñoz-Torres, Juan Ramón (2002): Por qué interesan las noticias. Un estudio de los fundamentos del interés informativo. Barcelona, Herder.

Navarro, Lizy (2009): “Tres lustros del periodismo digital: interactividad e hipertextualidad [Fifteen Years of Online Journalism. Interaction and Hypertextuality]".Comunicar, 33, 35-43. DOI: 10.3916/c33-2009-02-003.

Obregón, Rafael; Arroyave, Jesús; y Barrios, Marta (2010): "Periodismo y comunicación para la gestión de riesgo en la subregión andina: discursos periodísticos y perspectivas para un enfoque prospectivo y preventivo". Folios, 23, 105-135, en: http://goo.gl/ZWLhrx [Recuperado: 8 de agosto de 2012]

Piñuel, José Luis; Gaitán, Juan Antonio; y Lozano, Carlos (2013): Confiar en la prensa o no. Un método para el estudio de la construcción mediática de la realidad. Salamanca, Comunicación Social. 
Quesada, Montserrat (2007): Periodismo de sucesos. Madrid, Síntesis.

Rizo, Marta (2006): “Intersubjetividad, Vida Cotidiana y Comunicación”. Razón y Palabra, 57, en: http://goo.gl/D8KyXq [Recuperado: 18 de abril de 2013]

Rizo, Marta (2009): “Sociología fenomenológica y comunicología: Sociología Fenomenológica y sus aportes a la comunicación interpersonal y mediática". Revista Fronteiras, 11(1), 25 32, en: http://goo.gl/YQR5W1 [Recuperado: 1 de junio de 2013]

Román Portas, Mercedes (2000): “Aspectos metodológicos de la historia de la comunicación”. Ámbitos, 5, 119-128, en: http://goo.gl/So2EqV [Recuperado: 13 de septiembre de 2014]

Soria, Carlos (1997): El laberinto informativo: una salida ética. Navarra, Eunsa.

Tallón, José (1992): Lecciones de empresa informativa. Madrid, Ediciones Ciencias Sociales. UNESCO (1983): Código Internacional de Ética Periodística, en: http://goo.gl/R7Bxpw [Recuperado: 11 de febrero de 2015]

UNISDR [Estrategia Internacional para la Reducción de Desastres de las Naciones Unidas] (2009): Terminología sobre reducción del riesgo de desastres. Ginebra, en: http://goo.gl/ LfxSug [Recuperado: 11 de enero de 2012]

Urchaga, José David (2009): “Análisis de contenido”, en Reyes, R. (Coord.): Diccionario crítico de Ciencias Sociales. Madrid-México, Universidad Complutense \& Plaza-Valdés.

Villalobos, Margarita (1998): "Uso de los medios de comunicación en la prevención de desastres". Biblio-des, 26, 6-8, en: http://goo.gl/kJFO8q [Recuperado: 7 de agosto de 2013]

Wimmer, Roger \& Dominick, Josehp (1996): La investigación cientifica de los medios de comunicación. Una introducción a sus métodos. Barcelona, Bosch.

Wright, Charles (1972): Comunicación de masas. Buenos Aires, Paidós.

Silvia Alende Castro es doctora por la Universidad de Vigo (departamento de Psicoloxía Evolutiva e Comunicación de la Facultade de Ciencias Sociais e da Comunicación de Pontevedra). Su tesis Periodismo de Prevención en Galicia. El concepto de comunicación útil en la prensa diaria tuvo la calificación de Sobresaliente Cum Laude.

Silvia Alende Castro es doctora por la Universidad de Vigo (departamento de Psicoloxía Evolutiva e Comunicación de la Facultade de Ciencias Sociais e da Comunicación de Pontevedra). Su tesis Periodismo de Prevención en Galicia. El concepto de comunicación útil en la prensa diaria ha recibido recientemente la cualificación de Sobresaliente Cum Laude.

Aurora García González es doctora en Comunicación Pública y Profesora Titular de Periodismo en la Facultad de Ciencias Sociales y de la Comunicación de la Universidad de Vigo. Es autora, entre otros, de: Manual del Comunicador radiofónico (Barcelona, 2001) y Escribir y hablar en los medios (LEA 2005)

José David Urchaga Litago es doctor y Profesor de la Universidad Pontificia de Salamanca (España) de las materias de Psicología, Estadística y Metodología de la Investigación. Sus investigaciones se centran en análisis de contenido, estadística multivariante y psicología positiva. 